\title{
On the physical system modelling of energy storages as equivalent circuits with parameter description for variable load demand (Part I)
}

\author{
Cong-Toan Pham and Daniel Månsson
}

\begin{abstract}
Energy storages take a key role in electrical energy balancing in our power grid in respect to the increasing utilization of renewable energies. Assessing the effectiveness of energy storages and finding the optimal use under varying load conditions is essential which requires accurate modeling. This study develops equivalent circuit models for different energy storages. The model parameters $R, L, C$ and $U_{b}$ define the storage system in question allowing us to analyze storage devices under varying load conditions. Energy storages feature non-linear characteristics which are reflected in variable model parameters.
\end{abstract}

Index Terms-Batteries, capacitors, equivalent circuits, energy storage, flywheels, ultracapacitors, pumped hydro storage, smart grid.

\section{INTRODUCTION}

$\mathrm{T}$ HE Smart Grid of the future envisions a reliable, sustainable and environmental friendly power supply. To achieve this goal renewable energy sources, e.g., wind and photovoltaic power will replace fossil fueled power plants causing greater unpredictable power generation. One way of compensating is balancing any demand and supply mismatch through the use of energy storage systems (ESS). The effectiveness of specific energy storages has been studied for various scenarios in for example wind power integration, load management and power quality etc. [1, 2]. The assessment of energy storages to find their optimal use and placement in grid applications is an essential step. This involves the evaluation of technical, economic, environmental and social aspects of the ESS against each other. Multi-criteria selection methods such as Fuzzy Logic and Analytic Hierarchy Process has been suggested as an initial assessment tools [3, 4] weighting selected storage parameters against each other. The technical requirements are the first and most important aspects to determine the suitability of ESS. One of the crucial technical requirements is the response time of ESS; or in other words how fast the ESS can adapt to changes of power demand. In practice energy storages are accompanied with ancillary equipment, which are usually subjected to certain technical limitations such as maximum allowed current or maximum

This Project is financed by STandUP for Energy.

C.T. Pham is with the Department of Electromagnetic Engineering, Royal Institute of Technology (KTH), Stockholm, Sweden, e-mail: (ctpham@kth.se)

Prof. Daniel Månsson is with the Department of Electromagnetic Engineering, Royal Institute of Technology (KTH), Stockholm, Sweden motor power. Determining the technical suitability requires indepth understanding of the technical limits, in this case the physical background and the dynamic behavior of ESS. Models help to quantify the attributes of physical systems in parameters, allowing us to describe their relations in mathematical expressions. Fortunately, most physical systems exhibit recurrent and similar governing laws, which make it possible to compare different systems through key parameters.

This paper aims to introduce a general equivalent circuit model applicable on various ESS for analyzing varying loads. The circuit parameters comprise the physical attributes relevant to the dynamic behavior, including non-linear effects which we present in the following sections for each of the following storage type (capacitor, flywheel, battery and pumped hydro station). The chosen approach is also modular and compatible with existing storage models, which can be incorporated into this model.

In the upcoming sections we will discuss the similarities between different physical systems (Section II.1) and use that fact to develop a representative electrical circuit model for energy storages with electrical components (Section II.2). This model should be able to analyze various power demands which is further described under section II.3. From section III onwards the circuit model approach is applied to four different ESS types (capacitor, flywheel, battery and pumped-hydro station) including the description of the relevant circuit components based on the original physical system.

\section{EQUIVALENT CIRCUIT MODEL}

\section{II.1 Analogy of physical systems}

All processes involved in physical systems occur with the exchange of energy, including the conversion between different energy types (chemical, thermal, electrical etc.). The capability of a system to perform work to another depends on the energy state both systems exhibit relatively to each other. The way how the systems interact relies on the physical properties which influence the transfer rate of energy [5], e.g. the temperature difference between two objects is the driving force of the energy transfer and vanishes when the temperature difference becomes zero. How fast this process is depends on the boundary separating both systems, i.e. the specific thermal conductivity and geometrical dimensions of the wall. 
TABLE I. ANALOGY BETWEEN PHYSICAL QUANTITIES

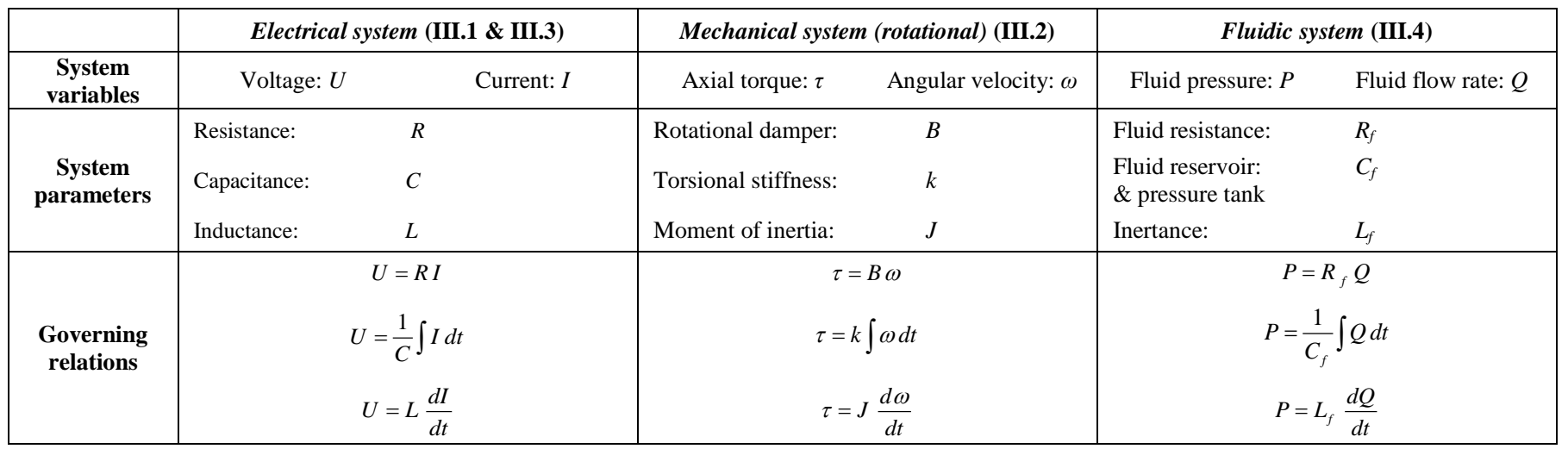

This same fundamental concept of energy transfer allows us to design an unifying model which can describe various physical systems [5].

The equivalent circuit method refers to a theoretical representation of the physical properties of a physical system (electrical, mechanical, thermal etc.), usually to simplify the complex characteristics into more convenient and clearly distinguishable components. This concept has been useful in many engineering areas retaining all physical characteristics in circuit elements (e.g. resistance $R$, spring constant $k$ etc.) which still describe the system's dynamics accurately $[5,6]$. All physical systems relate power and energy by the product of their system variables, similar to the power and work done in kinematics $\left(P=F v, W=\int F v \mathrm{dt}\right)$ or electrical systems $(P=U I$, $\left.W=\int U I \mathrm{dt}\right)$. Note, that the abbreviations of system variables and parameters can be similar, especially in this paper where we compare parameters of different physical systems, e.g., capacitance $C$ and fluid capacitance $C_{f}$. Reference [5] denotes the system variables as effort $(e)$ and flow $(f)$, which represent the extensive (e.g., voltage, force, temperature etc.) and intensive (e.g., current, velocity, heat flow etc.) property, respectively. The analogy can also be found in the governing relations of the system variables as shown in Table. I. The losses and parameter description of the individual systems is summarized in TABLE II. The system elements portray the properties of the physical systems, e.g., the electrical resistance limits the electric current passing through a conductor (Ohm's law) which is comparable with friction reducing the velocity of a moving object. Another example is Newton's second law of motion $(F=m d v / d t)$ which similarly links the relation between the voltage and current of an inductor $(U=L d I / d t)$. Due to this analogy the parameters in each physical system directly translate to each other (e.g., resistance $R \equiv$ friction $B$; capacitance $C \equiv$ inverse spring constant $1 / \mathrm{k}$; inductance $L \equiv$ mass $m$ ). In circuit theory these elements are usually constant, but this assumption is far from reality as each element can be dependent on further variables, e.g. time or temperature. For example, in fluidic systems the fluid resistance $R_{f}$ is a function of the flow rate $Q$, resulting in a non-linear relation $\left(p=R_{f}(Q) Q\right)$.

\section{II.2 Mathematical derivations}

In this study we focus on the storage media itself (flywheel, electrochemical cell, pressure tank etc.) including the essential components influencing the charging and discharging dynamics. The energy inserted into the ESS will be either stored as kinetic (through an inductance $L$ ) or potential energy (through a capacitance $C$ or a voltage source $U_{p o t}$ ). In the circuit representation the energy storages can either be charged by a voltage or current source, which leaves us with four possible circuits. One circuit with a voltage source is presented in Fig. 1 consisting of two parts: 1a) illustrates the kinetic storage model, whereas $1 \mathrm{~b}$ ) shows the potential storage part. The input to the potential storage model in this case is set by a dependent current source which is determined by the kinetic model. For example, in a pumped-hydro station the flow rate $Q$ to fill or empty the upper reservoir (potential storage) needs time to build up and depends on the transported fluid mass. If the build-up time for the current is sufficiently fast the kinetic part of the storage model can be safely neglected, i.e. a low inductance resulting in a small time constant $(\tau=L / R \rightarrow 0)$. With given circuit components $R$ and $L$ the dynamic behavior of the kinetic model is characterized by the following ordinary differential equation (ODE).

$$
U_{i n}=L \frac{d I(t)}{d t}+R I(t)
$$

The left side of the equation describes the input voltage causing the change of current. The solution to (1) with a stepup excitation of $U_{i n}$, as later described in II.3, is an exponential function.

$$
I(t)=I_{0} e^{-\frac{t}{L / R}}+\frac{U_{i n}}{R}\left(1-e^{-\frac{t}{L / R}}\right)
$$

This expression is applicable for all kinetic ESS with $R$ representing the loss terms: e.g., electrical resistances in superconducting magnetic energy storages or drag friction in flywheels etc. Another loss term can be included on the right hand side of (1) as a voltage source $U_{\text {loss }}$ countering the input voltage, e.g., describing static forces which needs to be overcome first. This loss term only affects the charging phase for cases where $U_{\text {in }}>U_{\text {loss }}$; otherwise if $U_{\text {in }}<U_{\text {loss }}$ applies $U_{\text {in }}$ is set to 0 as if no input is present. 
Kinetic storage



(a)



(b)

Fig. 1. Equivalent circuit models for kinetic and potential storages with voltage source input. a) kinetic energy storage. b) potential energy storage.

The $R C$-branch characterizes the dynamic behavior of the potential storage model. For the potential storage part the transient response solely relies on the time constant $\left(\tau=R_{p} C\right)$ branch. The input current $I_{\text {in }}(=I(t))$ divides into $I_{r}$ and $I_{c}$.

$$
I(t)=I_{\text {in }}(t)=I_{c}(t)+I_{r}(t)
$$

Replacing $I_{c}=C d U_{c} / d t$ and $I_{r}=U_{c} / R_{p}$ results in (4).

$$
I_{\text {in }}(t)=C \frac{d U_{c}(t)}{d t}+\frac{U_{c}(t)}{R_{p}}
$$

To solve the non-homogenous ODE in (4) we derive the homogenous $\left(y_{h}\right)$ and particular $\left(y_{p}\right)$ solution with $y_{h}+y_{p}$ equal to the full solution. The homogenous solution is the solution with no input source, which is simply an exponential function similar as in (2) with $\tau=R_{p} C$ instead. The particular solution can be found in standard reference for exponential inputs.

$$
\begin{gathered}
U_{c}(t)=y_{h}(t)+y_{p}(t) \\
y_{h}(t)=K e^{-\frac{t}{R_{p} C}} \\
y_{p}(t)=\frac{\left(I_{0}-\frac{U_{i n}}{R}\right)}{C\left(-\frac{R}{L}+\frac{1}{R_{p} C}\right)} e^{-\frac{t}{L / R}+\frac{R_{p}}{R} U_{i n}}
\end{gathered}
$$

With the initial condition $U_{c}(0)=U_{0}$ and $t=0$ we can solve for the coefficient $K$ and achieve the full solution using (5-7).

$U_{c}(t)=\left[U_{0}-\frac{\left(I_{0}-\frac{U_{i n}}{R}\right)}{C\left(-\frac{R}{L}+\frac{1}{R_{p} C}\right)}-\frac{R_{p}}{R} U_{i n}\right] e^{-\frac{t}{R_{p} C}}+\frac{\left(I_{0}-\frac{U_{i n}}{R}\right)}{C\left(-\frac{R}{L}+\frac{1}{R_{p} C}\right)} e^{-\frac{R_{t}}{L}}+\frac{R_{p}}{R} U_{i n}$

Expression (8) presents the general solution considering the transient part for the current increase. If the time constant in (1) is negligible ( $\tau=L / R \rightarrow 0$ ), we can simplify the current input as a step-up function. This is safe to assume for batteries and capacitors, where the current can be immediately set. With this assumption (8) can be reduced to (9).

$$
U_{c}(t)=U_{0} e^{-\frac{t}{R_{p} C}}+R_{p} I_{i n}\left(1-e^{-\frac{t}{R_{p} C}}\right)
$$

The expressions (1-9) apply to the charge, discharge and idle operation with some assumptions for each operation. In discharge mode the voltage and current inputs will be reversed by a minus sign $-I_{\text {in }}$ and $-U_{\text {in }}$ in all expressions (1-9). In idle mode all inputs are set to zero $\left(I_{i n}=0\right.$ and $\left.U_{i n}=0\right)$ leaving only the homogenous solution of (1).

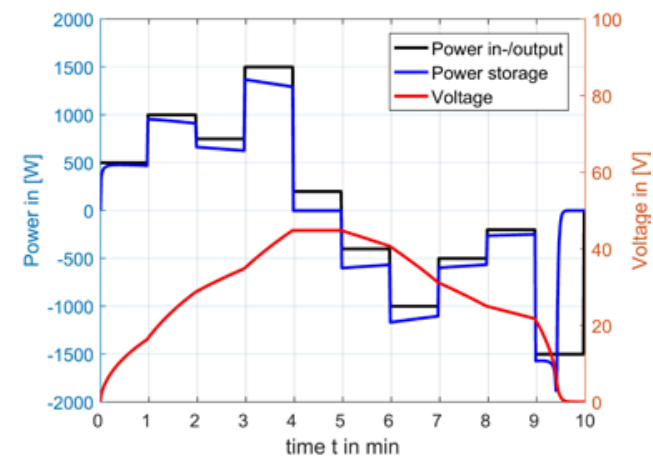

Fig. 2. Example of a power profile with minute sampling. During the time step $\Delta t$ the power in-/output is assumed to be constant. At each time step the end values for voltage are transferred to the next time step as initial values.

$$
I(t)=I_{0} e^{-\frac{t}{L / R}}
$$

Expression (10) however still feeds in (4); an exponentially declining input resulting in (11).

$$
U_{c}(t)=\left[U_{0}-\frac{I_{0}}{C\left(-\frac{R}{L}+\frac{1}{R_{p} C}\right)}\right] e^{-\frac{t}{R_{p} C}}+\frac{I_{0}}{C\left(-\frac{R}{L}+\frac{1}{R_{p} C}\right)} e^{-\frac{t}{L / R}}
$$

If $\tau=L / R$ approaches zero then (11) reduces to (12).

$$
U_{c}(t)=U_{0} e^{-\frac{t}{R_{p} C}}
$$

For a static potential storage the voltage remains constant, but the amount of charges defines the total energy absorbed or delivered.

$$
Q_{b}(t)=Q_{0}+\int I_{\text {in }} d t
$$

With the expressions so far we can now calculate the amount of energy stored or lost in each circuit element. As mentioned earlier the available energy in an ESS is stored through the “capacitor" $E_{c}$, "inductor" $E_{l}$ or "voltage source” $E_{b}$, which we can calculate as followed.

$$
\begin{aligned}
& E_{c}(t)=\frac{C}{2}\left(U_{c}(t)\right)^{2} \\
& E_{l}(t)=\frac{L}{2}(I(t))^{2} \\
& E_{b}(t)=U_{p o t} Q_{b}
\end{aligned}
$$

The losses are characterized by the resistances $R$ and $R_{p}$.

$$
E_{\text {losses }}(t)=R \int I^{2}(\mathrm{t}) d t+\frac{1}{R_{p}} \int U_{c}^{2}(t) d t
$$

\section{II.3 Implementation of fluctuating load conditions}

Time variation plays an important role as the ESS are demanded to adapt to the changes immediately. Furthermore, the circuit parameters can also vary with time or other variables. Implementing those in our expressions is tedious and alternatively we suggest a more direct approach. Input data is usually sampled at fixed time-steps (seconds, hours etc.). Information about charging or discharging demand is only available at the time a sample is measured, leaving us with no specific information in between these time steps. We therefore simplify and assume a step up change and constant parameters for each time step. With this assumption we can 
apply our equations (1-12) at each time step $\Delta t$ individually. For the consecutive time steps the end values of the previous time step are used as initial conditions with $j$ as the number of the current sample time $(j=1 \ldots \mathrm{k})$ (see Fig.2).

$$
\begin{aligned}
& I_{0, j+1}=I_{l}\left(t_{j}+\Delta t\right) \\
& U_{0, j+1}=U_{c}\left(t_{j}+\Delta t\right) \\
& Q_{0, j+1}=Q_{b}\left(t_{j}+\Delta t\right)
\end{aligned}
$$

To consider the non-linear effects the circuit parameters can also change at each time-step which will be discussed in the following sessions. The accuracy of the model hence increases with smaller time steps as the changes per time step decrease.

Voltage and current data can be directly used as inputs, but in cases where only a power profile is available the approach differs. The total input power $P_{\text {in }}$ is divided among the circuit components; each power calculated by the product of voltage and current at the respective component (e.g., $P_{c}(t)=U_{c}(t) I_{c}(t)$ ) at each time-step.

$$
P_{i n}\left(t_{j}\right)=\sum_{m=1}^{n} P_{m}\left(t_{j}\right)
$$

In between the time steps we assume the power in- and output to be constant (Fig. 2). For example, using the expressions for the potential storage model the total power of the circuit elements equals the input power and to achieve that depends on the input current $I_{\text {in }}$.

$$
P_{i n}\left(t_{j}\right)=P_{b, j}\left(\Delta t, I_{i n}\right)+P_{c, j}\left(\Delta t, I_{i n}\right)+P_{r, j}\left(\Delta t, I_{i n}\right)
$$

The input current is iteratively approximated to match with the input power at each time $t_{j}$. In case of power demand $\left(P_{\text {out }}\right)$ the power drawn from the storage equates to the desired output power plus the losses.

$$
P_{\text {out }}\left(t_{j}\right)+P_{r, j}\left(\Delta t, I_{\text {out }}\right)=P_{b, j}\left(\Delta t, I_{\text {out }}\right)+P_{c, j}\left(\Delta t, I_{\text {out }}\right)
$$

How well the storage follows the reference power depends on the time-constant of the individual storage. With higher resistance values less energy will be stored and more energy needs to be invested to achieve the demanded output.

\section{ENERGY STORAGE MODEL}

In the following sections we will apply the equivalent circuit model approach on various ESS including capacitors, batteries, flywheels and pumped-hydro storages.

\section{III.1 Capacitor and Ultracapacitors}

Capacitors are classified as electrical storage devices as they store energy in form of electric charges (potential energy). The appropriate circuit is described by (9) and Fig. 3. An usual setup consists of two or more metallic plates/foils separated by a dielectric material with low electrical conductivity, i.e., high resistance $R_{p}$. Ideally, the potential increases linearly to the amount of charges inserted multiplied by a proportionality factor $\left(U_{c}=Q C\right)$. This factor is the capacitance $C$ (in $\mathrm{F}$ ) which defines the charge capacity a capacitor can store per unit of voltage. $C$ is in large a geometrical quantity, described by shape, size, distance of the conductive plates, and the permittivity $\varepsilon_{r}$ of the dielectric material [7]. The capacitance can be derived from Gauss's law of electrostatics and for parallel plates $C$ takes the form of

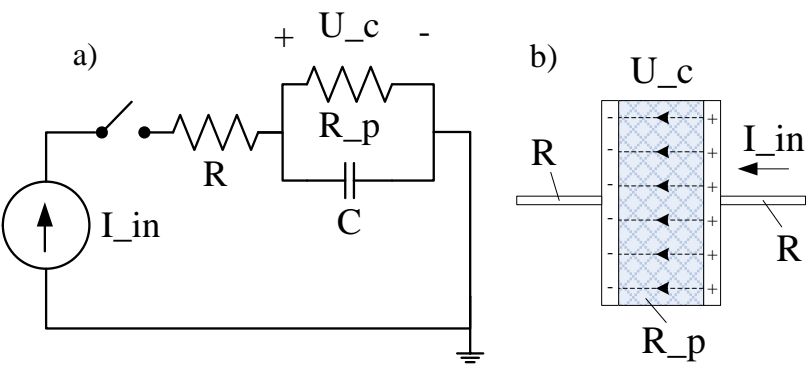

Fig. 3. a) Equivalent circuit models for capacitors/Ultracapacitors,

b) simplified structure of a capacitor

Parallel plates: $\quad C=\varepsilon_{r} \varepsilon_{0} \frac{A}{d}$

where, in case of the parallel plates, $A$ is the surface area of one plate and $d$ the distance between them [7]. The actual behavior of a capacitor is also frequency dependent, which is seen at high frequency excitations (above $f>1 \mathrm{Mhz}$ [8]), but in our case we concentrate on load management $(d P / d t>1 \mathrm{~s})$ rather than on power quality applications $(d P / d t<1$ s). With the assumption of slow paced changes and if none of the geometrical conditions change during the operation the capacitance can be considered constant.

Electrical double-layer capacitors (Ultracapacitor) differ from normal capacitors by using a liquid or solid electrolyte instead of a solid dielectric material. This type of capacitors then also displays electrochemical properties using the polarization of the electrolyte as an additional capacitive storage. This enhances the total charge capacity per unit area, thus increasing the energy density. The electrical double-layer in the electrolyte behaves similar to a pure capacitor and features same linear characteristics $\left(I_{c}=C d U / d t\right)$ [9]. Determining the capacitance through the electrical doublelayer is mathematically challenging to comprise into a simple circuit representation as in Fig. 3. A more direct approach has been suggested by [10] to determine the equivalent circuit parameters experimentally. The total capacitance measured will be the combination of both effects derived from Gauss's law and the polarization of the electrolyte. One method is to measure the change in stored charges relative to the change of the capacitor's potential in a time frame $t_{1}$ to $t_{2}$.

$$
C=\frac{\Delta Q}{\Delta U}=\frac{\int_{t_{1}}^{t_{2}} I(t) d t}{\left(U_{1}-U_{2}\right)}
$$

The parallel resistance $R_{p}$ (from Fig.3) can be calculated by the capacitor's self-discharge characteristics. With a starting potential $U_{1}$ we let the capacitor discharge to a certain potential level $U_{2}$ while measuring the time to reach to that point.

$$
U_{2}=U_{1} e^{-\frac{\Delta t}{R_{p} C}}
$$

Solving (26) for $R_{p}$ yields

$$
R_{p}=-\frac{\Delta t}{C \ln \left(U_{2} / U_{1}\right)} .
$$

The total series resistance can be estimated with $R=L /\left(\sigma_{c} A_{c}\right)$ if the conductors properties are known (length $L$ in m, electrical conductivity $\sigma_{c}$ in $\mathrm{S} / \mathrm{m}$, cross-section area $A_{c}$ in $\mathrm{m}^{2}$ ). 

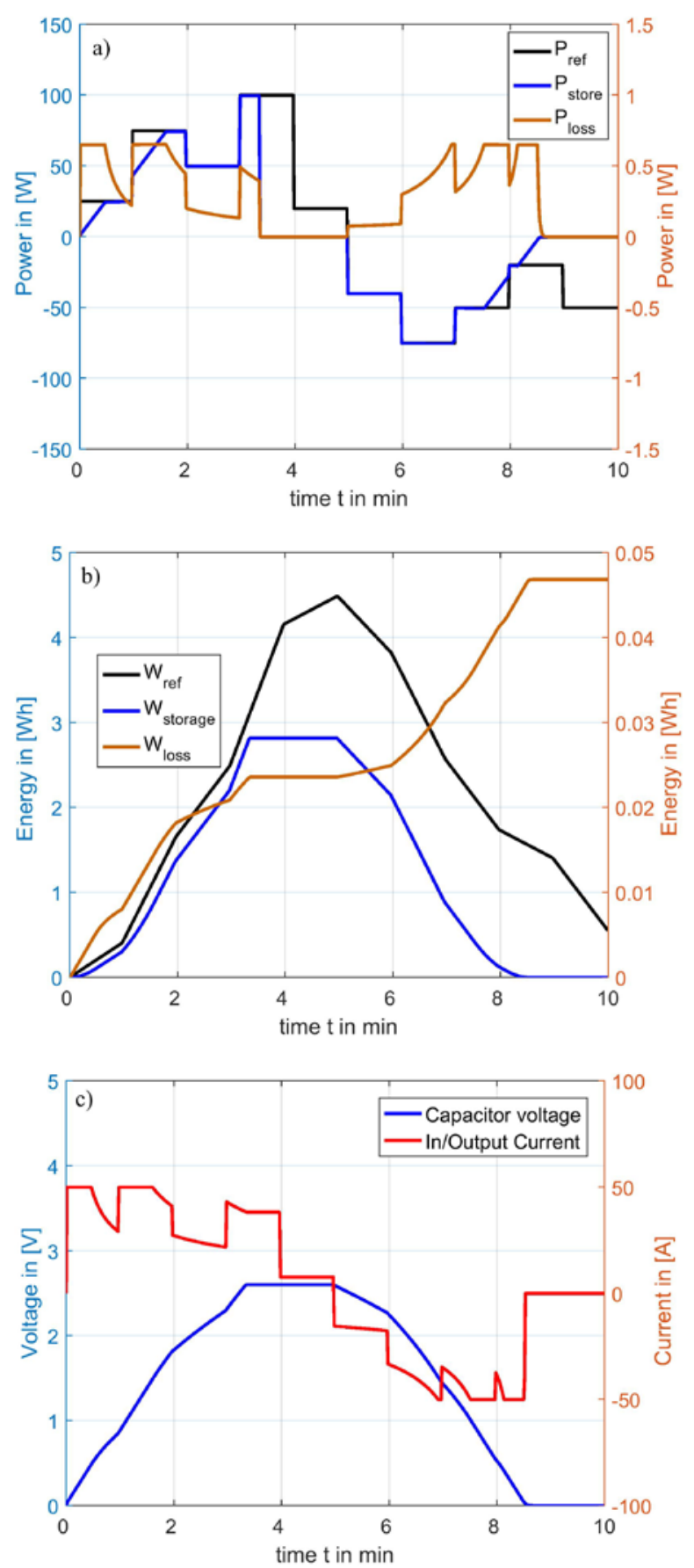

Fig. 4. Charge \& discharge profile for an ultracapacitor $\left(\mathrm{V}_{\max }=2.6 \mathrm{~V}\right.$, $\mathrm{R}=0.26 \mathrm{~m} \Omega, \mathrm{R}_{\mathrm{p}}=500 \Omega, \mathrm{C}=3000 \mathrm{~F}$ ). a) Power profile of the storage compared to the reference power. b) Storage capacity (max. $2.81 \mathrm{Wh}$ ) compared to the reference energy. c) Voltage and current profile (max. 50A)

Alternatively, $R$ can also be measured through a voltage and current change using Ohm's law. At the moment a step-up voltage change $\Delta U$ is imposed on the circuit all voltage will first apply at $R$ with a measurable current spike $\Delta I$ [10].

$$
R=\frac{\Delta U}{\Delta I}
$$

Fig. 4 exemplarily shows the charging and discharging characteristic for a small commercially available ultracapacitor with $2.6 \mathrm{~V}$ rating. In this case we can limit the maximum current to $50 \mathrm{~A}$, if for example the cables can handle only that much. With the given specifications the storage mostly manages to follow the demanded power in- and output (Fig. 4a) until the maximum capacity at 2.6V (Fig. 4c), calculated after (14), is reached. An initially empty capacitor requires much higher input current to match with the required input power following the relation of $P=I U$, reversely a capacitor will also discharges much faster with greater depth of discharge. Due to our current restriction the peak currents are cut off as seen in Fig. 4c near the starting and ending time. In this parameter setup the losses are low leading to high system efficiencies around 99\%; note though, that we only consider an ideal capacitor medium not the usually attached ancillary system with its own losses. With this, the model approach presented here allows us to analyze energy storages with technical limitations and track their dynamic behavior to various conditions.
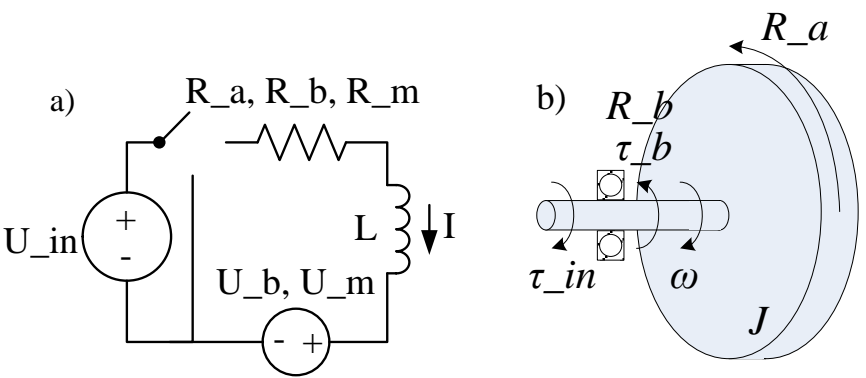

Fig. 5. a) Equivalent circuit model for flywheel b) simplified structure of a flywheel

\section{III.2 Flywheel Energy Storage System (FESS)}

A FESS comprises of a flywheel rotor connected to a motor/generator unit. The motor imposes a torque onto the flywheel causing a change in rotational speed. A suspension system is needed to absorb the occurring dynamic and static loads due to the vibrations and weight of the system [11].

Bearings are usually used to cushion those loads, but on the other hand reduce the input torque due to friction (e.g., static, coulomb friction). Another loss component is the aerodynamic drag which increases with higher rotor velocity [12]. The dynamic behavior of the FESS is then characterized by the flywheel rotor mass and the friction components.

The energy is stored through the rotation of the rotor mass. Our system variables $U$ and $I$ directly translate to the torque $\tau$ (Nm) applied and angular velocity $\omega(\mathrm{rad} / \mathrm{s})$. The inductance $L$ in our circuit represents the moment of inertia $J$. Here, the kinetic storage model in Fig. 5 and (2) apply for the FESS. Geometrical and material properties define $J$ which is in general described by the volume integral over the mass in respect to the distance from the rotation axis. For most cases the rotor takes the shape of a wheel rim which simplifies our expression to $J=k m r^{2}$ [13]; with the shape factor $k$ (e.g., wheel rim $k=0.5$ ), the mass $m$ (in $\mathrm{kg}$ ), and radius $r$ (in $\mathrm{m}$ ). Otherwise $J$ can also be calculated by using tensile strength $\sigma=\rho r^{2} \omega_{\max }^{2}$ [13]. Replacing the radius with $\sigma$ yields

$$
L \equiv J=m r^{2}=\frac{\sigma}{\omega_{\max }^{2}} \frac{m}{\rho} .
$$

Expression (29) has the advantage that the limit of the flywheel system is given by the maximum angular velocity. 
For values below this threshold we can assume a rigid body that $L$ remains constant in the operation range. The friction forces on the other hand cannot be assumed constant as they change with the rotor speed. The derivation of the friction components, expressed as torque losses, originates from thermodynamics and fluid mechanics with [11], [12] providing a comprehensive description of the main losses in flywheel systems.

To calculate the aerodynamic drag requires the flywheel geometry and properties. According to [12] the aerodynamic drag $\left(\tau_{a}\right)$ is a non-linear function of the density of the medium surrounding the flywheel (usually air) $\rho_{g}$ in $\mathrm{kg} / \mathrm{m}^{3}$, the rotor radius $r$ in $\mathrm{m}$, and the dimensionless drag coefficient $C_{m}$ : The drag losses are reflected as $R_{a}$ in Fig. 5.

$$
\tau_{a}=\rho_{g} r^{5} C_{m} \omega^{2}=R_{a}(\omega) \omega
$$

Using air as the surrounding medium the density can be estimated through the ideal gas law (pressure $p$ in $\mathrm{Pa}$, universal gas constant $R=8.315$ in $\mathrm{J} / \mathrm{molK}$, molar mass of gas $M_{m}$ in $\mathrm{kg} / \mathrm{mol}$, temperature $T$ in $\mathrm{K}$ ).

$$
\rho_{g}=\frac{p M_{m}}{R T}
$$

$C_{m}$ is dimensionless coefficient which is a function of further coefficients; the Reynolds number $R_{e}$ and Knudsen number $K_{n}$.

$$
\begin{aligned}
& \operatorname{Re}=\rho_{g} r^{2} \omega / \eta \\
& K_{n}=\lambda / r
\end{aligned}
$$

The Reynolds number is defined as the ratio between the inertial forces (density $\rho$ ) and viscous forces (dynamic viscosity $\eta$ ) of the fluid in motion (see, e.g., [12]). Dependent on which force is dominant the flow pattern behaves either laminar $\left(R_{e}<5 \cdot 10^{4}\right)$, turbulent $\left(R_{e}>5 \cdot 10^{4}\right)$ or in between both stages (transition $R_{e} \approx 5 \cdot 10^{4}$ ) [12]. It is an important key parameter in fluid dynamics which effectively describes the state of flow, and greatly affects the friction losses.

The Knudsen number relates the average distance a molecule travels without collision with another molecule to a representative length (radius) of the flywheel. A high value for the mean free path $\lambda$ indicates a low presence of molecules; or in other words vacuum condition. Calculating $C_{m}$ analytically has proven to be extremely difficult, but literature provides empirical expressions with sufficient accuracy in the designated flow regions. Dependent on the Knudsen number different expressions have been proposed. For $K_{n}<1$ the drag torque coefficient for constant thickness discs can be approximated with ([12]):

$$
\begin{aligned}
& C_{m}=3.87 \mathrm{Re}^{-1 / 2} \\
& \left(\operatorname{Re}<5 \cdot 10^{4}\right) \\
& C_{m}=0.146 \mathrm{Re}^{-1 / 5} \\
& \left(\operatorname{Re}>5 \cdot 10^{4}\right) \text {. }
\end{aligned}
$$

The Knudsen number becomes important for flywheels spinning in free air with sufficient space to a surrounding enclosure. Reducing the gap between the flywheel and the housing, lower than the thickness of the boundary layer [12], will result in the following two formulae.

$$
C_{m}=2.67 \mathrm{Re}^{-1 / 2} \quad\left(\operatorname{Re}<3 \cdot 10^{5}\right) .
$$

$$
C_{m}=0.0622 \mathrm{Re}^{-1 / 2} \quad\left(\operatorname{Re}>3 \cdot 10^{5}\right) .
$$

Combining (34-37) with (30) then yields the drag resistance $R_{a}$ for four different cases.

$$
R_{a}(\omega)=\rho_{g} r^{5} C_{m} \omega
$$

For $K_{n}>10$ we enter the region of high vacuum (130 $\mathrm{Pa}>p$ ) where the following approximation for drag losses has been proposed [12].

$$
\tau_{a}=\rho_{g} r^{4}\left(\frac{2 K T N_{a}}{M_{m}}\right)^{-1 / 2} \omega
$$

In the transition region between $0.1<K_{n}<10$ the flow neither behaves as a continuous flow or free molecule stream. This region is difficult to describe mathematically.

The second contributor to losses is the friction from the bearing connection with the rotor shaft. Various types of bearings exist, but ball and magnetic bearings are most common in FESS [11]. The drag torque of ball bearings is caused by material hysteresis, friction between adjacent parts and viscous drag of the used lubricant. At high speed operation the first two causes are dominant compared to viscous drag in low speed operation [11]. A first estimate, only valid under normal operating conditions and with good lubrication, is provided by [14], where $\mu$ is a constant coefficient of friction and $P_{1}$ the equivalent dynamic bearing load. Furthermore, $P_{1}$ differs with the type of bearings used, expressed as the basic dynamic load rating $C$ which can be found in the product tables from [14].

$$
\tau_{b}=0.5 \mu d_{b} P_{1} \quad\left(P_{1} \approx 0.1 \mathrm{C}\right)
$$

For greater accuracy the friction losses can be calculated individually for each bearing by the sum of the rolling, sliding, seals and drag losses [14].

Magnetic bearings offer the advantage of providing suspension without direct contact and lubrication. This is advantageous in vacuum systems as no lubrication can diffuse into the flywheel chamber, which is one of the main reasons why magnetic bearings are typically seen in vacuum operated systems instead. An initial estimation for the drag torque has been suggested for a five active axis suspension system with $b$ as the number of poles of the bearing, the gravitational acceleration $g$, and the flywheel mass $m$ [11]. The combination of (41) and (42) yields the total bearing losses in the system.

$$
\begin{aligned}
& \tau_{m, 1}=m g\left(3.2 \times 10^{-5}\right) \\
& \tau_{m, 2}=1.3 \times 10^{-8} b \omega=R_{m} \omega
\end{aligned}
$$

The aerodynamic and bearing losses can be equally expressed as circuit elements; as $R_{a}$ stands for the aerodynamic losses (38), so does $\tau_{b}$ for the constant bearing losses (40) as an opposing voltage source ( $U_{b}$ in Fig. 5). To account for the non-linearity the values for each element will be updated at each time-step. The end-value of one step-up input, here $\left(I_{j-1}\left(t_{j}\right)=I_{0, j}\right)$, at the time $t_{j}$ serves as the input value for the new $R_{a, j}\left(I_{0, j}\right)$ and $U_{b, j}\left(I_{0, j}\right)$. The torque losses in magnetic bearings behave linearly, divided into a resistive $\left(R_{m}=m g 1.3 \cdot 10^{-8} \mathrm{~b}\right)$ and an opposing source part $\left(U_{m}=m g 3.2 \cdot 10^{-5}\right)$. 

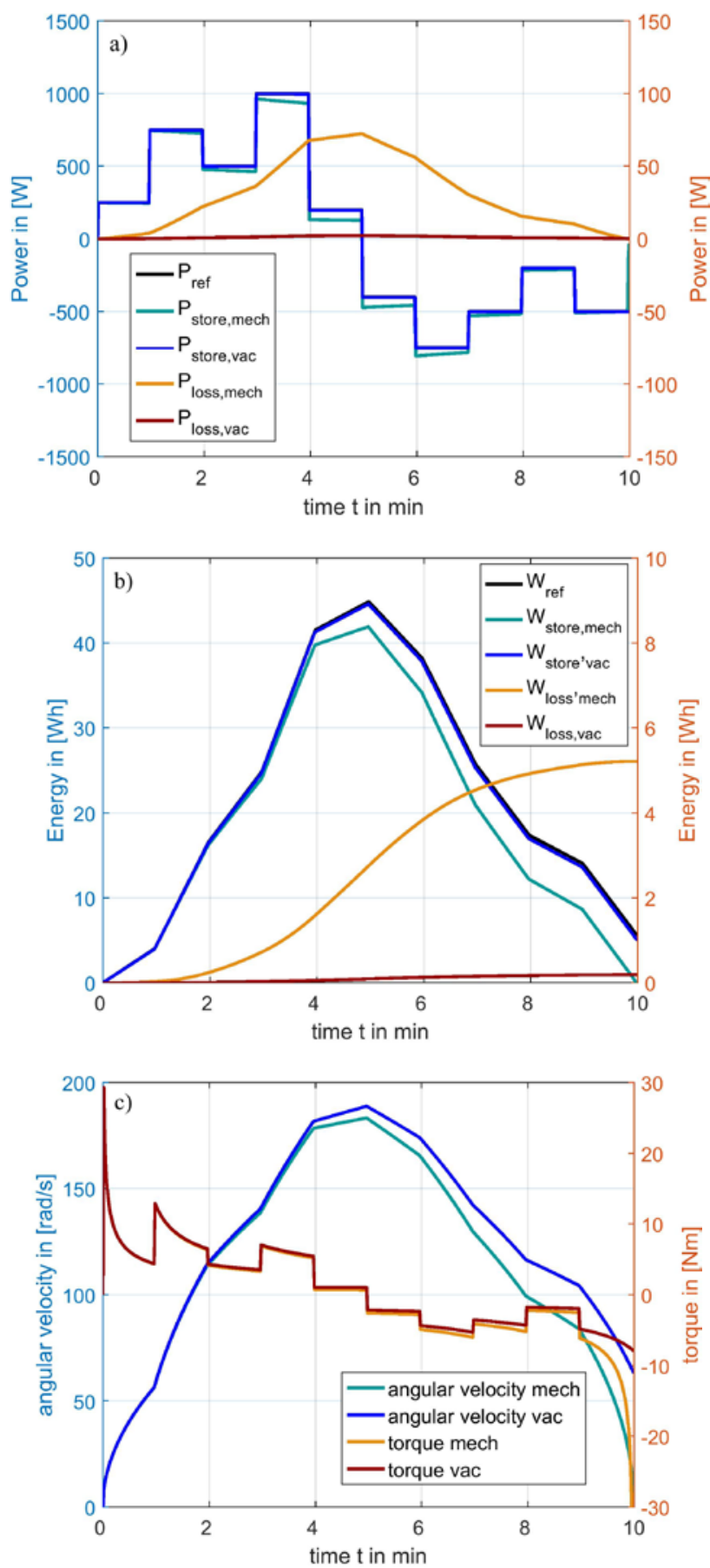

Fig. 6. Charge \& discharge profile for two flywheels $\left(J=9 \mathrm{kgm}^{2}\right)$. Flywheel "vac": vacuum operated flywheel with magnetic bearings; Flywheel "mech": ambient pressure operated flywheel with ball bearings a) Power profile of flywheels compared to the reference power. b) Flywheel capacities compared to the reference energy. c) Angular velocity and torque profile of both flywheels in comparison

In Fig. 6 two different flywheel systems are compared to each other with the same power imposed on them. The vacuum operated system clearly stands out in terms of minimal losses. The flywheel with mechanical bearings features increasing losses with higher velocity, resulting in a much faster discharge of the capacity (see Fig. 6a \& Fig 6b). This is also clearly visible in the power profile where the power input is deviating from the reference.
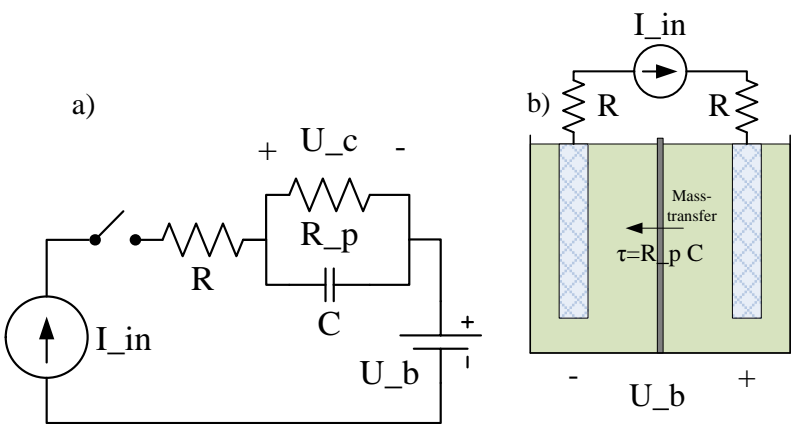

Fig. 7. a) Equivalent circuit model for batteries b) simplified structure of a battery cell

\section{III.3 Battery Energy Storage System (BESS)}

Batteries store electrical energy by electrochemical conversion into chemical energy using galvanic cells. The three main components of a battery are the electrodes (anode \& cathode) and the electrolyte. The speed of the electrochemical processes in the battery cell defines the dynamic behavior. There has been great effort of predicting and understanding battery behavior accurately via mathematical and empirical models. The electrochemical processes are complex and difficult to describe without indepth knowledge of the cell design including the electrolyte, the shape and material of the electrodes [15] etc. Hence, for engineering purposes empirical models are more often employed which portray the dynamics satisfactorily accurate $[14,15]$. The most common model representation is the circuit diagram as depicted in Fig. 7, consisting of a voltage source, inner resistance and a $R_{p} C$-branch. The main storage element is the voltage source $U_{b}$ instead of the capacitive component $C$ which portrays the mass transfer limitations [15].

The main driving force for charge transfer is the potential difference between the electrodes, referred to as the open circuit voltage (OCV), $U_{b}$ in Fig 7 , of the battery cell. The Nernst equation [15] relates the OCV to the electrode materials and the actual operation condition of the battery cell, where $R_{g}$ is the gas constant $\left(R_{g}=8.315 \mathrm{in} \mathrm{J} / \mathrm{mol} \mathrm{K}\right), T$ the temperature in Kelvin, $n$ the number of electrons involved in the stoichiometric reaction, and $F$ the Faraday's constant $(\approx 96.5000 \mathrm{C} / \mathrm{mol})$ (43). $E^{0}$ is the potential difference between the standard potentials of the electrode materials with no reaction taking place. Under operation the surface concentrations of the active species ( $c_{o x d}$ concentration of oxidized species \& $c_{\text {red }}$ concentration of reduced species) changes at the respective electrodes (anode \& cathode). Dependent on the relation between the concentrations the overall cell voltage will either increase or decrease [15].

$$
U_{b}=U_{\text {pot }}=E^{0}+\frac{R T}{n F} \ln \left(\frac{c_{\text {oxd }}}{c_{\text {red }}}\right)
$$

Using the surface concentrations for calculating the OCV is rather inconvenient as it requires further mathematical descriptions of the concentration changes during operation. A more convenient way is to express (43) in dependence of the state-of-charge (SOC), proposed by [17] for lithium intercalation electrodes. 

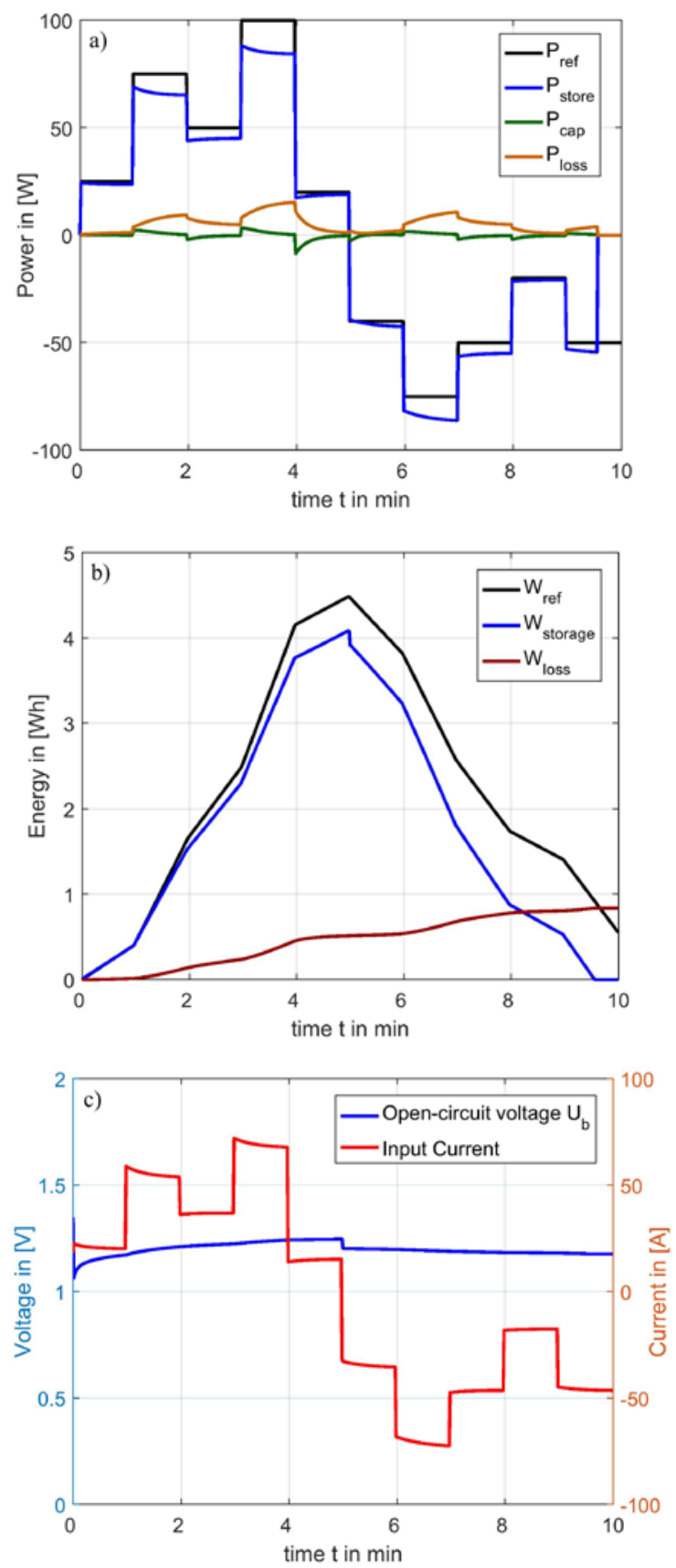

Fig. 8. Charge \& discharge profile for NiMH battery cell (85Ah, $1.35 \mathrm{~V}_{\text {nom, }}$ $\mathrm{R}=0.78 \mathrm{~m} \Omega$ ). a) Power profile of NiMH compared to the reference power. b) NiMH capacity compared to the reference energy. c) Current in- and output of the battery cell; Change of open circuit voltage over time

Discharge: $U_{p o t, D}=E_{D}^{0}+\frac{R T}{n F} \ln \left(\frac{S O C_{s}}{1-S O C_{s}}\right)+\sum_{k=1}\left(v_{k, \mathrm{D}}\right)\left(S O C_{s}\right)^{k}$

Charge: $\quad U_{p o t, \mathrm{C}}=E_{C}^{0}+\frac{R T}{n F} \ln \left(S O C_{s}\right)+\sum_{k=1}\left(v_{k, \mathrm{C}}\right)\left(S O C_{s}\right)^{k}$

The coefficients $v_{k}$ are determined experimentally to fit in the actual OCV of the selected battery type. The index $s$ under SOC denotes a small correction due to the use of surface concentrations instead of the bulk concentration. $k_{m}$ is the mass transport coefficient and $Q_{0}$ the initial capacity [17].

$$
S O C_{s}=S O C-\frac{I}{k_{m} Q_{0}}
$$

The anode collects the free electrons released during the electrochemical process and transfers them through an external circuit to the cathode, where the electrons recombine with oxidized ions. The flow of electrons in this case is limited by the resistances the electrode contacts and the conductor. Both effects are governed by Ohm's law where the conductor resistance is a function of its geometry. The contact resistance of the electrodes consists of the sum of electrical conductivities of each component in proportion to the total electrode with the cross-section area of $A_{e}$ [15].

$$
R=\frac{L}{\sigma_{c} A_{c}}+\frac{1}{A_{e} \sum_{k} x_{k} \sigma_{k}}
$$

The second process to influence the overall electrochemical reaction is the mass transport of ions from and to the electrodes. The mass transfer of ions is the result of the electrical driving force due to electrode potentials (see OCV) and diffusion through a concentration gradient. The movement of ions due to the electric field has a linear relation similar to Ohm's law using $\kappa$ as an equivalent electrical conductivity for the electrolyte, whereas the diffusion mechanisms need further derivation of Fick's law of diffusion [15]. The diffusion is mainly the difference of concentration of the electroactive species at the electrode surface compared to the bulk concentration. With ongoing process the concentrations will slowly equalize reducing the driving force of the mass-transfer [15]. Investigating the transport mechanisms in electrolytes is complex and has been developed by [18] as an electric circuit representation. However, their circuit analogy of mass transport exceeds our modest approach in complexity, which would require much more circuit elements. Note though, that the proposed storage models can always be further expanded, i.e. by introducing more components to describe the storage's behavior more accurately if needed. Alternatively, some studies extracted the characteristic parameters (here $R_{p}$ and $C$ ) through parameter estimation methods and experimental observation [17], [16]. Ref [17] relates $R_{p}$ through the following expression with the activation energy $E_{\mathrm{A}, \mathrm{R}}$.

$$
R_{p}=\left(\sum_{k=0}^{n j}\left(r_{k}\right)\left(S O C_{s}\right)^{k}\right) e^{\left(\frac{-E_{A, R}}{R T}\right)}-R
$$

The time constant can be determined by short pulse current experiments [17]. The battery response then follows the behavior according to (9) with a step-up input. With known time constant $C$ can be easily found by $C=\tau / R_{p}$.

Fig. 8 illustrates the power and energy profile for a NiMHbattery. The effective storage of energy only concerns the electrode potential and its change shown in Fig. 8c. The losses are not only affiliated to the ionic and ohmic losses in the circuit $\left(P_{\text {loss }}\right.$ in Fig 8a), but also to the capacitance which accumulate charges reducing the actual amount of charges reaching the electrodes. Furthermore, a higher requested output power further increases the actual power drawn from the storage as the losses rise exponentially with the current $I$ through the series resistance $R\left(P=R I^{2}\right)$. Thus, the storage cannot provide the demanded power at a certain power threshold where the losses will surpass the usable power. 




Fig. 9. a) Equivalent circuit model for pumped-hydro storages b) simplified structure of a pumped-hydro station

\section{III.4 Pumped-hydro Storage (PHS)}

Pumped-hydro storages count as hydro electrical storages, which store potential energy by pumping water from a lower height to an upper height reservoir (see Fig. 9). With water as the energy carrying medium PHS are usually dealt as fluidic systems, using pressure $p(\mathrm{~Pa})$ and volumetric flow rate $Q$ $\left(\mathrm{m}^{3} / \mathrm{s}\right)$ as system variables [5]. The circuit in Fig. 9 is then defined by (2), (9), (11) and (12).

The main components of a PHS are the upper reservoir, the turbine/pump and the water pipeline. The PHS model divides into a kinetic and a potential storage part. The kinetic part describes the inertia of the water flow rate, i.e. when the pump imposes a torque onto the fluid mass and how fast the mass is then accelerated. The constitutive relation for the acceleration of mass originates from Newton's second law of motion $(F=m d v / d t)$ [5]. In a fluidic system this results in (49), where pressure is the driving force for the acceleration.

$$
p_{L}=\frac{\rho l_{p}}{A_{p}} \frac{d Q_{L}}{d t}=L_{f} \frac{d Q_{L}}{d t}
$$

The inertance $L_{f}$ is the equivalent to the inductance $L$ and is a function of the pipe's geometry ( $l_{p}$ length of pipe, $A_{p}$ crosssection area in $\mathrm{m}^{2}$ ). Under normal operation water behaves as an incompressible medium, i.e. with the density $\rho$ remaining constant $L_{f}$ stays constant. The flow rate follows an exponential growth as described in (2) and feeds in the potential storage part. Unlike in the battery model the capacitor is the main storage unit in addition to the potential gain from the height of the reservoir. The water level increase of the reservoir is comparable with the potential increase in a capacitor; its fluid capacitance (equal to $C$ in Fig. 9) defined by the reservoirs area $A_{r}$, the gravitational acceleration $g$, and the water density $\rho$.
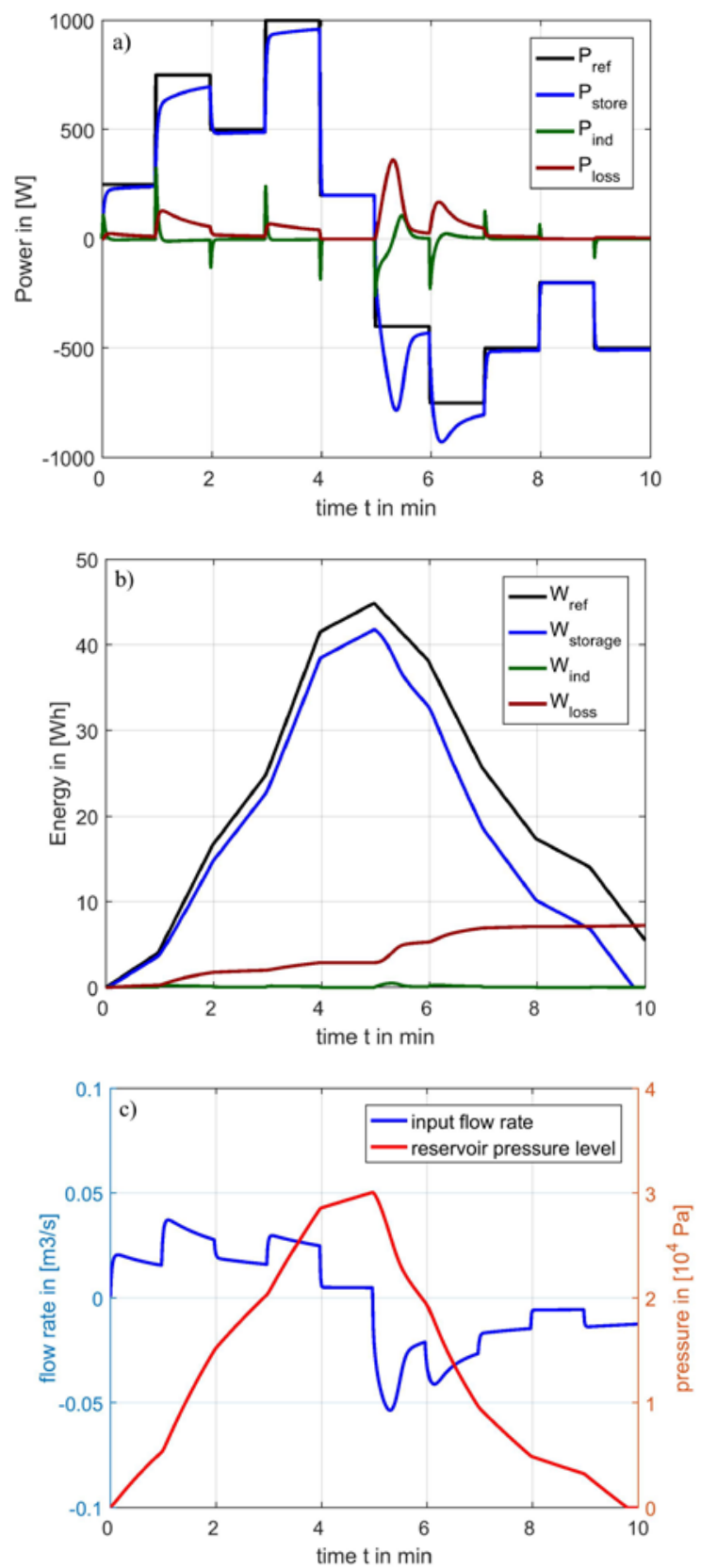

Fig. 10. Charge \& discharge profile for a PHS (Pipe: $l_{p}=10 m, d_{p}=0.1 \mathrm{~m}$, $\mathrm{L}_{\mathrm{f}} \approx 1.2710^{6} \mathrm{~kg} / \mathrm{m}^{4}$; open reservoir: $\mathrm{A}_{\mathrm{r}}=2 \mathrm{~m}^{2}, \mathrm{C}_{\mathrm{f}} \approx 210^{-4} \mathrm{~m}^{5} / \mathrm{N}, \mathrm{h}=1 \mathrm{~m}$ ). a) Power profile of PHS compared to the reference power. b) PHS capacity compared to the reference energy. c) Flow rate and pressure of the PHS

$$
Q_{C}=\frac{A_{r}}{\rho g} \frac{d p_{C}}{d t}=C_{f} \frac{d p_{C}}{d t}
$$

Self-discharge losses (leakage, evaporation) in PHS are negligible [1], thus it is safe to assume an infinitely large $R_{p}$. The height of the reservoir is expressed as an additional hydrostatic pressure gain, represented by $U_{b}$ in Fig. 9 [19].

$$
U_{b} \equiv p_{b}=\rho g h
$$

With an incompressible medium the fluid capacitance, inertance and hydrostatic pressure are constant.

The only variable component is the drag loss in the pipeline. For a circular shaped pipe the pressure lost is calculated by (52), with $l$ the length, $d$ the diameter, and $A_{p}$ the cross-section 
area of the pipe. The model equivalent resistance $R$ is the in parenthesis enclosed expression.

$$
p_{R}=R_{f} Q=\left(\lambda(\operatorname{Re}) \frac{\rho}{2} \frac{l}{d A^{2}} Q\right) Q
$$

The friction factor $\lambda$ is an empirical dimensionless factor which relates the pressure loss to the flow pattern [19]. Similar to the drag losses in FESS the flow can either be laminar or turbulent using the Reynold's number [19].

$$
\operatorname{Re}=\frac{\rho d Q}{A \eta}=\frac{4 \rho Q}{\pi d \eta}
$$

In a laminar flow $\lambda$ is estimated through the Hagen-Poiseuille equation (54). For higher Reynold numbers $\lambda$ can only be estimated through iteration by Colebrook-White [19]. Beside $\operatorname{Re}$ the roughness $\varepsilon$ of the pipe material also influences the flow and needs to be included in the expressions, especially in turbulent flows. The equation listed here (54-56) are valid for hydraulic smooth pipes [19].

$$
\begin{array}{ll}
\lambda=\frac{64}{\operatorname{Re}} & (\operatorname{Re}<2300) \\
\frac{1}{\sqrt{\lambda}}=1.14-2 \log \left(\frac{\varepsilon}{d}\right) & (2300<\operatorname{Re}<500 \\
\frac{1}{\sqrt{\lambda}}=-2 \log \left(\frac{2.51}{\operatorname{Re} \sqrt{\lambda}}+0.27 \frac{\varepsilon}{d}\right) & (\operatorname{Re}>5000)
\end{array}
$$

A high value of inertance results in a slow buildup (high $L / R$ ) of the necessary flow rate into the reservoir, indicated by the power spikes of $P_{k i n}$ in Fig. 10a and the more rounded curvature of the flow rate in Fig. 10c. This inertia will delay the energy in- or output of the main storage unit and can become critical to follow for high fluctuating power demand. The pipe design is crucial for achieving high efficiencies of the PHS as the losses increase with higher flow rate (best seen at $\mathrm{t}=6 \mathrm{~min}$ in Fig 10a). In the worst case the PHS cannot deliver the demanded power output if the losses are too high. This is an indicator for a flawed pipe design, e.g. if the pipe diameter is too small for high flow rates.

\section{CONCLUSION}

This paper introduced an equivalent circuit model for various types of energy storages applicable for varying power demand. We showed that the different energy storages, here capacitors, batteries, flywheels and PHS, are a derivation of the general circuit model. The circuit representation captures the main physical properties of ESS and its dynamic behavior represented by the time constants. The circuit elements can be derived or experimentally determined from the corresponding energy storage type. Non-linear characteristics are also considered with the assumption that the circuit elements are kept constant during a time interval and then change with the initial condition for the consecutive time step. The modularity of this approach is advantageous as the accuracy of the model can be further increased by adding more circuit elements. Real applications have technical limits, e.g. motor with maximum power or cables with maximum allowed current flow. These limitations can be incorporated into our model which allows us to analyze and assess more realistic ESS for different power

\begin{tabular}{|c|c|c|}
\hline Type & Formulae & Comments \\
\hline \multirow{3}{*}{ Capacitor } & $C=\frac{\Delta Q}{\Delta U}=\frac{\int_{t_{1}}^{t_{2}} I(t) d t}{\left(U_{1}-U_{2}\right)}$ & $\begin{array}{l}\text { Measure start \& end } \\
\text { voltage } U_{1}\left(t_{1}\right) \& U_{2}\left(t_{2}\right) \text {. } \\
\text { Measure current } I \text { during } \\
\text { time interval. }\end{array}$ \\
\hline & $R=\frac{\Delta U}{\Delta I}$ & $\begin{array}{l}\text { Step-up voltage input. } \\
\text { Measure current change } \\
\Delta I \text { with known } \Delta U \text {. }\end{array}$ \\
\hline & $R_{p}=-\frac{\Delta t}{C \ln \left(U_{2} / U_{1}\right)}$ & $\begin{array}{l}\text { Self-discharge. } \\
\text { Measure start \& end } \\
\text { voltage } U_{1}\left(t_{1}\right) \& U_{2}\left(t_{2}\right) \\
\text { during time interval. }\end{array}$ \\
\hline \multirow{6}{*}{ Flywheel } & $L=m r^{2}=\frac{\sigma}{\omega_{\max }^{2}} \frac{m}{\rho}$ & $\begin{array}{l}\text { Geometrical and material } \\
\text { properties known. }\end{array}$ \\
\hline & $R_{a}=\rho_{g} r^{5} C_{m} \omega$ & $\begin{array}{l}\text { Aerodynamic drag in air } \\
(\mathrm{p}>130 \mathrm{~Pa}) \text {. } \\
\mathrm{C}_{\mathrm{m}} \text { changes with } \\
\text { rotational speed and } \\
\text { flywheel design. }\end{array}$ \\
\hline & $R_{a}=\rho_{g} r^{4}\left(\frac{2 K T N_{a}}{M_{m}}\right)^{-0.5}$ & $\begin{array}{l}\text { Aerodynamic drag in air } \\
(\mathrm{p}<130 \mathrm{~Pa}) .\end{array}$ \\
\hline & $R_{m}=1.3 \times 10^{-8} b$ & $\begin{array}{l}\text { Magnetic bearing linear } \\
\text { losses. }\end{array}$ \\
\hline & $U_{b}=0.5 \mu d_{b} P_{1}$ & Ball bearing loss. \\
\hline & $U_{m}=m g\left(3.2 \times 10^{-5}\right)$ & Magnetic bearing loss. \\
\hline \multirow{5}{*}{ Battery } & $\begin{array}{l}U_{b, D}=E_{D}^{0}+\frac{R T}{n F} \ln \left(\frac{S O C_{s}}{1-S O C_{s}}\right) \\
+\sum_{k=1}\left(v_{k, \mathrm{D}}\right)\left(S O C_{s}\right)^{k}\end{array}$ & $\begin{array}{l}\text { OCV at discharge } \\
\text { changes with state-of- } \\
\text { charge (SOC). }\end{array}$ \\
\hline & $\begin{array}{l}U_{p o t, C}=E_{C}^{0}+\frac{R T}{n F} \ln \left(S O C_{s}\right) \\
+\sum_{k=1}\left(v_{k, C}\right)\left(S O C_{s}\right)^{k}\end{array}$ & $\begin{array}{l}\text { OCV at charge changes } \\
\text { with state-of-charge } \\
\text { (SOC). }\end{array}$ \\
\hline & $R=\frac{L}{\sigma_{c} A_{c}}+\frac{1}{A_{e} \sum_{k} x_{k} \sigma_{k}}$ & $\begin{array}{l}\text { Series resistance through } \\
\text { cables, connections, } \\
\text { electrodes }\end{array}$ \\
\hline & $\begin{array}{l}R_{p}=\sum_{k=0}^{n j}\left(r_{k}\right)\left(S O C_{s}\right)^{k} \times \\
e^{\left(\frac{-E_{A, R}}{R T}\right)}-R\end{array}$ & $\begin{array}{l}\text { Mass transfer limitations } \\
\text { of ionic charge travelling } \\
\text { between electrodes } \\
\text { changes with SOC. }\end{array}$ \\
\hline & $C=\tau / R_{p}$ & $\begin{array}{l}\text { Time constant } \\
\text { observation through short } \\
\text { pulse current experiment. }\end{array}$ \\
\hline \multirow{4}{*}{$\begin{array}{l}\text { Pumped- } \\
\text { hydro } \\
\text { station }\end{array}$} & $C_{f}=A_{r} / \rho g$ & $\begin{array}{l}\text { Geometrical parameters } \\
\text { of the reservoir. }\end{array}$ \\
\hline & $L_{f}=\rho l_{p} / A_{p}$ & $\begin{array}{l}\text { Geometrical parameters } \\
\text { of the connecting pipes. }\end{array}$ \\
\hline & $U_{b}=\rho g h$ & $\begin{array}{l}\text { Pressure gain due to } \\
\text { elevation. }\end{array}$ \\
\hline & $R_{f}=\lambda(\operatorname{Re}) \frac{\rho}{2} \frac{l}{d A^{2}} Q$ & $\begin{array}{l}\text { Pressure losses along the } \\
\text { pipes changes with flow } \\
\text { rate. }\end{array}$ \\
\hline
\end{tabular}

grid scenarios. The main parameters of each ESS type are listed in TABLE II.

TABLE II. LIST OF EQUIVALENT SYSTEM COMPONENTS FOR DIFFERENT ENERGY STORAGES 


\section{REFERENCES}

[1] X. Luo, J. Wang, M. Dooner, and J. Clarke, “Overview of current development in electrical energy storage technologies and the application potential in power system operation q,” Appl. Energy, vol. 137, pp. 511-536, 2015.

[2] O. Palizban and K. Kauhaniemi, "Energy storage systems in modern grids-Matrix of technologies and applications," J. Energy Storage, vol. 6, pp. 248-259, 2016.

[3] A. Barin, L. N. Canha, A. Da Rosa Abaide, and K. F. Magnago, "Selection of storage energy technologies in a power quality scenario - The AHP and the fuzzy logic," IECON Proc. (Industrial Electron. Conf., pp. 3615-3620, 2009.

[4] C. Pham and D. Månsson, "Suitability analysis of Fuzzy Logic as an evaluation method for the selection of energy storage technologies in Smart Grid applications,” 2015, pp. 452-457.

[5] P. E. Wellstead, Introduction to physical system modelling, Electronic. Control Systems Principles, 1969.

[6] E. T. Rolande, J. R. Albert, and J. T. Gregory, The Analysis \& Design of Linear Circuits, 7th ed. John Wiley \& Sons, Inc, 2012.

[7] D. J. Griffiths, Introduction to Electrodynamics, 4th ed. New Jersey: Prentice Hall, 2013.

[8] R. Thottappillil, "Electromagnetic compatibility (EMC).” KTH Royal Institute of Technology, Stockholm, 2014.

[9] R. A. Huggins, Energy Storage, 1st ed. Stanford, California: Springer, 2010.

[10] R. L. Spyker and R. M. Nelms, "Classical equivalent circuit parameters for a double-layercapacitor,” IEEE T. Aero. Elec. Sys., vol. 36, no. 3 Part 1, pp. 829-836, 2000.

[11] G. Genta, "Kinetic Energy Storage - Flywheel Suspension System," Kinet. Energy Storage, pp. 201-245, 1985.

[12] G. Genta, "Kinetic Energy Storage - The housing and vacuum system," Kinet. Energy Storage, pp. 169-200, 1985.

[13] A. G. Ter-Gazarian, Energy Storage for Power Systems, 2nd ed. IET, 2011

[14] SKF Group, “Rolling bearings,” 2012.

[15] T. B. Reddy, Linden's_handbo.pdf, 4th ed. Mc Graw Hill, 2011.

[16] J. Brand, Z. Zhang, and R. K. Agarwal, "Extraction of battery parameters of the equivalent circuit model using a multi-objective genetic algorithm,” J. Power Sources, vol. 247, pp. 729-737, 2014.

[17] M. W. Verbrugge and R. S. Conell, "Electrochemical and Thermal Characterization of Battery Modules Commensurate with Electric Vehicle Integration," J. Electrochem. Soc., vol. 149, no. 1, pp. A45A53, 2002.

[18] S. Rael and M. Hinaje, "Using electrical analogy to describe mass and charge transport in lithium-ion batteries," J. Power Sources, vol. 222, pp. 112-122, 2013.

[19] H. Schade and E. Kunz, Strömungslehre, 3rd ed. Walter de Gruyter, 2007.

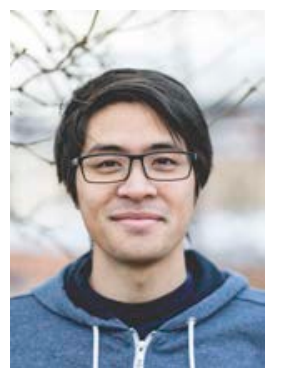

Cong-Toan Pham was born in Krefeld, Germany in 1987. He received his BEng degree from the Applied University of Duesseldorf, Duesseldorf, Germany and the MSc in Renewable energy \& energy efficiency at the University of Kassel, Kassel, Germany in 2014. Since 2014 has he been working towards his $\mathrm{PhD}$ in Electrical Engineering at the Royal Institute of Technology (KTH). His main research interest involves energy storages and the optimization of such in the power grid applications.

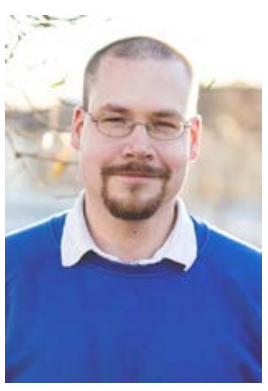

Daniel Månsson received his $\mathrm{MSc}$ and PhD in Engineering Physics in 2003 and 2008, respectively, from Uppsala University. His, PhD thesis was awarded the "2008 IET Sweden Network Prize for Student Excellence at Uppsala University" and one of the journal papers included was awarded the 2009 "Richard B. Schulz Best Transactions Paper Award” from the IEEE EMC Society. His research has focused on EMC and the threat of IEMI to civilian society. He is currently an associate professor at the Royal Institute of technology KTH working with Smart Electricity Grids and Power System Components. The research is, beside EMC, mainly focused towards different aspects of energy storage systems and their implementation. In academia, he is a member of the Swedish National Committee for Radio Science (SNRV), section E (Electromagnetic environment and interference) and, also, a member of the Swedish Physical Society. He teaches circuit analysis and electromagnetic compatibility and has received a pedagogical prize awarded by the students of the Master Programme in Engineering Physics; "Fjäder i hatten" for his teaching. In addition, he is a reviewer for the IEEE Transactions on Electromagnetic Compatibility, the PIER (Progress in Electromagnetic Research journals (regular, B, C and M)) and occasionally also for other journals. 